\title{
Cervical nucleoplasty as an effective treatment method of cervical degenerative disc disease
}

\author{
Yoo Sun Jung ${ }^{1}$, Seung Pyo Choi ${ }^{2}$, and Sung-Eun Sim ${ }^{2}$ \\ Department of Anesthesiology and Pain Medicine, ${ }^{1}$ Seoul National University Hospital, ${ }^{2}$ Seoul National University Boramae Hospital, \\ Seoul, Korea
}

Nucleoplasty is a minimally invasive spine intervention technique that uses controlled ablation, known as coblation to decompress the protruded intervertebral disc. Due to its short history and narrow indications, there is limited evidence concerning the efficacy and clinical outcome of cervical nucleoplasty. However, recent clinical experiences show that cervical nucleoplasty is a safe, convenient procedure with an excellent short-term clinical outcome.

A previously healthy 38-year-old male visited our clinic with numbness of the lateral part of his left lower arm and shoulder, tingling sensation and pain of his left thumb in which he has been suffering for 2 years. He showed no neurologic deficit. He rated his pain level as 8 out of $10 \mathrm{~cm}$ on the visual analogue scale (VAS). He received physical therapy and acupuncture for 2 years, but showed no improvement. Initial MRI study showed C4-5 disc extrusion, C5-6 disc protrusion and mild bulging of C6-7 disc (Figs. 1A-1D). He was scheduled for nucleoplasty of C5-6 disc. No analgesic medications were prescribed.

Prior to procedure, $1.0 \mathrm{~g}$ cefazolin was given intravenously as prophylaxis. Patient was placed on supine position on the operation table with his neck hyperextended. Vital signs were monitored and oxygen was supplied at $5 \mathrm{~L} / \mathrm{min}$ via nasal prong. The position and angle of the herniated part of the disc from the center of the disc was measured from the MRI. The C-arm was tilted obliquely to the opposite side of the patient's symptoms, according to the angle measured from the MRI. The needle entry point was marked on the right side of the patient at the center of the C5-6 disc level. The right internal carotid artery was displaced with the operator's fingers. The needle was advanced to the anterolateral annulus fibrosus. Local anesthetic ( $5 \mathrm{ml}$ of $2 \%$ lidocaine) was given from the disc to the skin, while repeated aspiration was performed.

Displacing the right internal carotid artery, a 19 G 3 inch introducer needle (ArthroCare Co., Sunnyvale, CA, USA) was advanced until it reached the annulus fibrosus of the C5-6 disc. The position of the needle tip was confirmed by the C-arm. Introducer needle was directed toward the center of the C5-6 disc to guide the estimated progression route under the oblique view of the C-arm. After withdrawing the stylet, the Perc ${ }^{\mathrm{TM}}$ DC Spine Wand ${ }^{\mathrm{TM}}$ (ArthroCare Co., Sunnyvale, CA, USA) was fastened to the needle hub. We advanced the introducer needle and Perc $^{\mathrm{TM}}$ DC Spine Wand ${ }^{\mathrm{TM}}$ to the estimated lesion. The Perc ${ }^{\mathrm{TM}}$ DC Spine Wand ${ }^{\mathrm{TM}}$ was connected to the Arthrocare system $2000^{\circledR}$ (ArthroCare Co., Sunnyvale, CA, USA), and coagulation was tested with the radio-frequency controller set for 1 to 2 seconds to check if there was movement or paresthesia in the patient's upper limbs. Checking the needle position by the lateral view of C-arm, coblation was performed for 15 seconds, while rotating the tip by 180 degrees, and repeated 3-4 times. Then, the tip was withdrawn a few milimeters, and repeated the same way above. The last coblation was performed when the tip of the needle was placed at the middle part of the disc in the lateral view of the Carm (Figs. 1E and $1 \mathrm{~F}$ ). The patient felt no discomfort during the procedure.

After the procedure, the patient took an absolute bed rest for four hours. One day after the procedure, the patient showed

Corresponding author: Sung-Eun Sim, M.D., Department of Anesthesiology and Pain Medicine, Seoul National University Boramae Hospital, 425, Sindaebang-dong, Dongjak-gu, Seoul 156-707, Korea. Tel: 82-2-870-2515, Fax: 82-2-870-1476, E-mail: euny62827@naver.com

() This is an open-access article distributed under the terms of the Creative Commons Attribution Non-Commercial License (http:// creativecommons.org/licenses/by-nc/3.0/), which permits unrestricted non-commercial use, distribution, and reproduction in any medium, provided the original work is properly cited. 

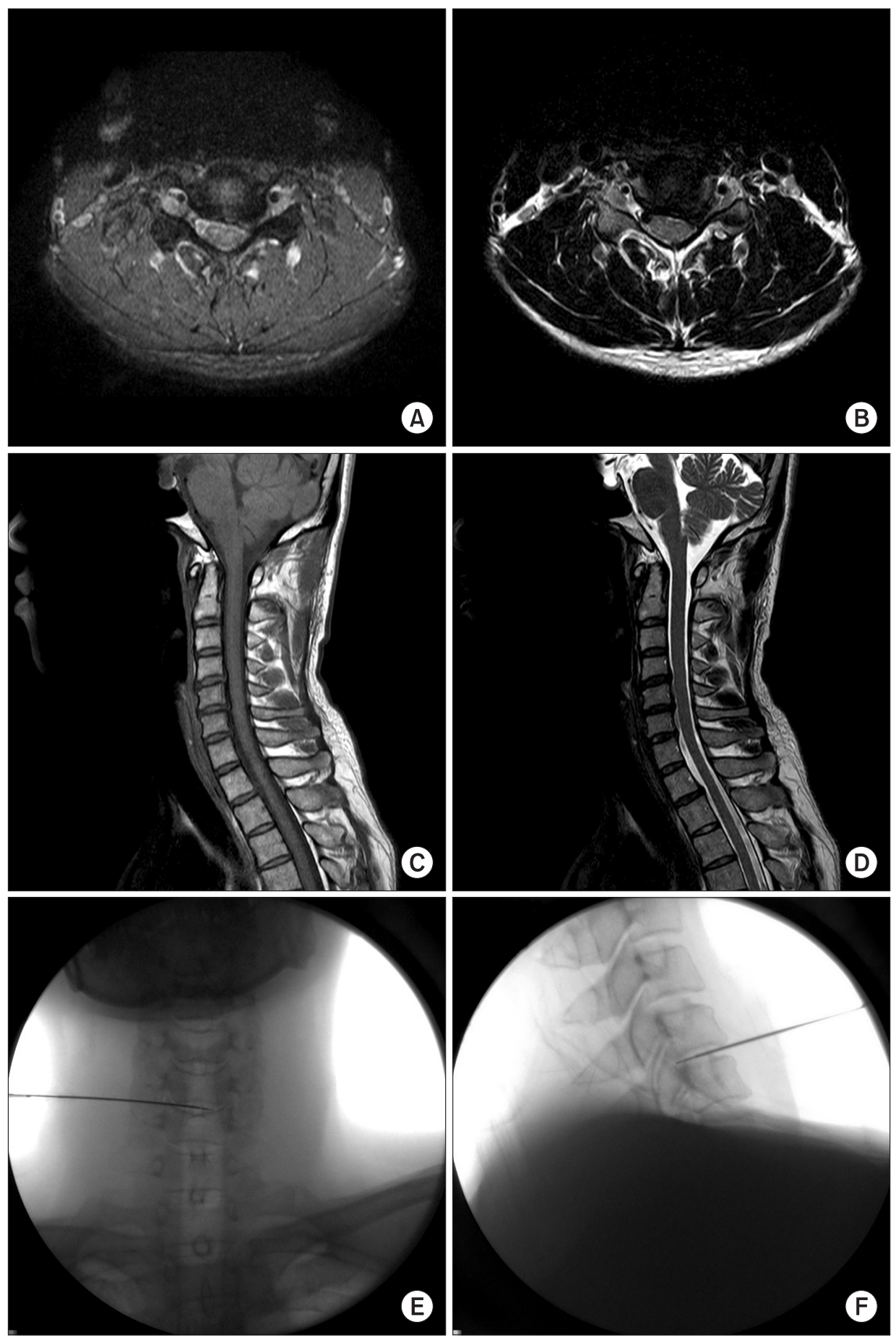

Fig. 1. Initial cervical spine MRI of the patient (A) Axial T1W image of C5-6 intervertebral disc (B) Axial T2W image of C5-6 intervertebral disc (C) Sagittal T1W image. (D) Sagittal T2W image. (E) The needle is placed at the C5-6 intervertebral space of the patient. AP view. (F) Lateral view.

improvement of numbness, tingling sensation and pain. Four weeks after the procedure, the patient visited our clinic for routine checkup and reported VAS score of 1 out of 10 without any numbness. The patient reported his satisfaction as 'good' according to the Modified Macnab criteria. There was no need for additional treatment.

Open surgery for cervical degenerative disc disease is an excellent treatment modality, but is invasive and requires long recovery period. Therefore, many patients and doctors seek for less invasive treatment modalities. Cervical nucleoplasty is a minimally invasive procedure to remove the protruded disc and relieve patients' symptoms. This procedure uses bipolar radiofrequency-based device, which functions via a plasma-mediated process to remove disc tissues. Strong electric fields are gener- 
ated at the electrode at the distal end of the device, and electrolytes, such as sodium ions in the nucleus pulposus, responds to the electric fields. With sufficient energy, the tissue around the electrode is vaporized and this ionized vapor (plasma field) can break molecular bonds in the adjacent tissue by chemical reactions. The organic molecules in the disc material (e.g. collagen) are easily converted to liquid and gas, and subsequently, desorbed from the targeted site. This results in reduction of tissue volume and relief of symptoms caused by compression with minimal histopathologic effect to the adjacent tissues [1].

Cervical nucleoplasty was first performed in South Korea at our center in 2007, and has shown successful results since then [2]. Indications for cervical nucleoplasty are limited [2-5]. Patients with lateral disc herniations, radicular/axial pain in which arm pain is more severe than neck pain, MRI evidence of contained herniation, and failure of conservative treatment, central herniation with axial neck pain that is unresponsive to 3 months of conservative treatment, and MRI evidence of contained her- niation and disc height more than $75 \%$ are candidates for cervical nucleoplasty [2].

In principle, if there is radiating pain during test stimulation, the tip should be withdrawn a few milimeters until there is no radiating pain. However, in some cases, radiating pain did not disappear, although we withdrew the tip to the posterior $1 / 3$ of the disc. There was negligible chance of direct stimulation of the nerve root or spinal cord in these cases. As such, we performed coblation regardless of the pain. In the end, there was no complication. We hypothesized that the radiating pain may be evoked by the nerve ending abnormally sprouting into the disc, but further research is needed, including histological examination.

In patients who do not respond to conservative treatment, cervical nucleoplasty should be considered as an effective treatment method. However, to be more conclusive, we should perform more studies and accumulate clinical data regarding the indications and the long-term results.

\section{References}

1. Bonaldi G, Baruzzi F, Facchinetti A, Fachinetti P, Lunghi S. Plasma radio-frequency-based diskectomy for treatment of cervical herniated nucleus pulposus: feasibility, safety, and preliminary clinical results. AJNR Am J Neuroradiol 2006; 27: 2104-11.

2. Sim SE, Ko ES, Kim DK, Kim HK, Kim YC, Shin HY. The results of cervical nucleoplasty in patients with cervical disorder : a retrospective clinical study of 22 patients. Korean J Pain 2011; 24: 36-43.

3. Ro JY, Oh CS, Kim OS, Kim DK, Woo NS, Shin HY. Nucleoplasty in a patient with cervical disc extrusion with radiating pain of an upper extremity. Anesth Pain Med 2009; 4: 203-7.

4. Birnbaum K. Percutaneous cervical disc decompression. Surg Radiol Anat 2009; 31: 379-87.

5. Calisaneller T, Ozdemir O, Karadeli E, Altinors N. Six months post-operative clinical and 24 hour post-operative MRI examinations after nucleoplasty with radiofrequency energy. Acta Neurochir (Wien) 2007; 149: 495-500. 